\title{
Experimental carotid occlusion: funduscopic and fluorescein angiographic findings
}

\author{
ALAN D. SPERTUS, JASON S. SLAKTER, SCOTT S. WEISSMAN, \\ AND PAUL HENKIND
}

From the Department of Ophthalmology, Montefiore Medical Center, Albert Einstein College of Medicine, Bronx, New York, USA

SUMMARY A characteristic fundus picture was consistently produced following acute bilateral common carotid artery ligation in mature rats, reminiscent of human carotid occlusive disease. Two days after ligation it consisted of dilatation and tortuosity of retinal veins, blurring and swelling of the optic disc, retinal whitening primarily along the venous distribution, and straightening of retinal arteries. Fluorescein angiography showed hyperfluorescence of the disc, delay in the rate of retinal arterial and venous filling, venous dilatation, disc oedema, disruption of the retinal capillary bed pattern, and late peripapillary staining/leakage. This pattern was not seen in rats which underwent acute unilateral ligation, although some mild changes were seen on fluorescein angiography. The vascular alterations seemed to regress spontaneously within one week. A peripapillary 'halo' and a granular-appearing nerve fibre layer developed later, exclusively in bilaterally ligated animals.

Neurologists and ophthalmologists have extensively reported the signs and symptoms of carotid artery disease in humans. ${ }^{1-6}$ The term 'venous-stasis retinopathy' was used by Kearns and Hollenhorst ${ }^{1}$ to characterise the appearance of the retinal venous circulation in carotid occlusive disease. It consisted of dilatation and tortuosity of the major retinal veins, microaneurysms, and small punctate haemorrhages. These findings were observed in approximately $5 \%$ of their patients with unilateral internal carotid artery occlusion or stenosis. They also reported sludging of blood within retinal veins in several of their patients with both carotid artery occlusion and an elevated sedimentation rate. Cotton-wool spots, ${ }^{23}$ retinal oedema,${ }^{34}$ pale optic discs with blurred margins, ${ }^{4}$ and retinal neovascularisation ${ }^{4}$ have also been reported in patients with carotid occlusion. David et al. ${ }^{7}$ reported a delay between the retinal arterial and venous phases of fluorescein angiography during digital carotid compression in human subjects. They found that slow venous filling, in the absence of venous obstruction, was most closely correlated with insufficiency of the retinal circulation. In another fluorescein angiographic study ${ }^{4}$ one patient with bilateral common

Correspondence to Paul Henkind, MD, Department of Ophhthalmology, Montefiore Medical Center, 111 East 210th Street, Bronx, New York 10467, USA. carotid occlusion was found to have late retinal venous leakage. In addition there was delay in arterial infilling of fluorescein dye and a gross delay in venous filling.

Methods for the detection of carotid occlusive disease have been studied utilising various animal

Table 1 Number of animals per subgroup division

\begin{tabular}{lllll}
\hline Group & I & II & III & IV \\
\hline A & 4 & 4 & 4 & 4 \\
B & 4 & 4 & 4 & 4 \\
C $_{1}$ & 2 & 2 & 2 & 2 \\
2 & 2 & 2 & 2 & 2 \\
\hline
\end{tabular}

A: Animals underwent unilateral common carotid artery ligation. B: Animals underwent bilateral common carotid artery ligations. $C_{1}$ : Animals underwent a sham unilateral common carotid artery ligation procedure.

${ }_{2}$ : Animals underwent a sham bilateral common carotid artery ligation procedure.

I: Animals underwent removal of ligation on day 8 ; enucleation and sacrifice on day 36 .

II: Animals underwent removal of ligation on day 29; enucleation and sacrifice on day 57.

III: Animals underwent removal of ligation on day 85 ; enucleation and sacrifice on day 113.

IV: Enucleation and sacrifice on day 113; no ligations removed.

Note: Group B animals had only 1 of 2 ligations removed on the dates above. Group $\mathbf{C}$ animals underwent a sham ligation removal procedure on the dates above. 
models. In one study ${ }^{8}$ a shoelace and a metal ring were used to ligate unilaterally a common carotid artery in rabbits. This model was used to examine alterations in intraocular pressure following the acute occlusion. The efficacy of ophthalmodynamometry and ocular pulse studies were evaluated in rabbits undergoing unilateral common carotid artery ligation. ${ }^{9}$ Ocular pulse studies proved more sensitive in detecting both acute and chronic carotid occlusion, particularly the latter. Fluorophotometry was performed in rabbits also subjected to unilateral common carotid ligation. ${ }^{10}$ A delay in fluorescein arrival in the fundus ipsilateral to the side of ligation was found. No difference in circulation time was noted between animals acutely and chronically occluded. Newsom et al. ${ }^{11}$ compared retinal fluorescein angiographic findings before and during common carotid occlusion in rhesus monkeys. They reported prolongation of the fluorescein passage through the retinal circulation in $86 \%$ of the animals studied.

Few animal studies have actually reported the pathological sequelae secondary to carotid occlusion. One such study involved unilateral and bilateral carotid ligation in mature gerbils. ${ }^{12}$ Approximately $1 / 3$ of the animals that underwent unilateral ligation had focal ischaemic damage to their brains and/or retinas by light microscopy. All of the bilaterally ligated animals died within 4 hours of surgery.

We have developed a model using pigmented rats which permits detailed examination of the retinal sequelae following both bilateral and unilateral carotid artery occlusion. Preliminary results of our study have been reported..$^{13}$ Here we analyse our fundus photograph and retinal fluorescein angiogram findings of the acute and chronic effects of carotid occlusion.

\section{Materials and methods}

Our experimental design called for the analysis of 48 Long-Evans pigmented female rats of at least 1 year of age. One hundred and twenty-six rats were needed to arrive at the final $\mathbf{4 8}$ study animals. Of the 126 rats, 30 were excluded prior to the initial operation due to the presence of dense cataracts. Thirtyfive died during the study as a result of anaesthesiaeither due to an initial and sudden respiratory arrest or, later, secondary to severe pulmonary congestion. Thirteen additional rats died during operative procedures, usually as a result of haemorrhage.

The animals were randomly divided into 3 experimental groups, undergoing $(a)$ unilateral or $(b)$ bilateral common carotid artery ligations, or $(c)$ sham ligation procedures to provide controls. They were further subdivided according to the length of time the ligations remained in place (Table 1). When an animal died during the study, its data was discarded and another rat substituted.

All animals underwent fundus photography and fluorescein angiography: (1) at the outset to establish baseline levels; (2) 2 days following initial operation; (3) immediately prior to ligation removal (if performed); (4) immediately prior to enucleation and sacrifice. In order to accomplish this they were deeply

Table 2 Percentage of eyes showing specific abnormalities by fundus photography*

\begin{tabular}{|c|c|c|c|c|c|c|c|c|c|c|}
\hline & \multicolumn{5}{|c|}{ Unilateral ligation } & \multicolumn{5}{|c|}{ Bilateral ligation } \\
\hline & $I$ & $I I$ & III & $I V$ & Total & $I$ & $I I$ & $I I I$ & $I V$ & Total \\
\hline \multicolumn{11}{|l|}{ Two days following ligation: } \\
\hline Venous dilation & 0 & 0 & 12 & $\mathbf{0}$ & 4 & 100 & 100 & 100 & $50 \dagger$ & 92 \\
\hline Arterial straightening & 25 & 0 & 0 & 0 & 4 & 88 & 100 & 83 & 100 & 92 \\
\hline Disc oedema & 0 & 0 & 0 & 0 & 0 & 75 & 88 & 100 & 75 & 85 \\
\hline Retinal whitening & 0 & 0 & 0 & 0 & 0 & 75 & 100 & 67 & 100 & 85 \\
\hline \multicolumn{11}{|l|}{ Prior to ligation removal: } \\
\hline Venous narrowing & 0 & 0 & 12 & - & 6 & 33 & 25 & 50 & - & 36 \\
\hline Arterial narrowing & 0 & 0 & 0 & - & 0 & 33 & 12 & 12 & - & 18 \\
\hline Arterial dilatation & 0 & 25 & 25 & - & 19 & 33 & 62 & 50 & - & 50 \\
\hline Increased light reflex & 0 & 25 & 12 & - & 12 & 17 & 62 & 100 & - & 64 \\
\hline Granular nerve fibre layer & 0 & 0 & 0 & - & 0 & 33 & 75 & 100 & - & 73 \\
\hline Peripapillary halo & 0 & 0 & $\mathbf{0}$ & - & 0 & 33 & 75 & 88 & - & 68 \\
\hline \multicolumn{11}{|l|}{ Prior to sacrifice: } \\
\hline Venous narrowing & 0 & 0 & 0 & 0 & 0 & 0 & 29 & 67 & 43 & 35 \\
\hline Arterial narrowing & 0 & 0 & 0 & 0 & 0 & 50 & 14 & 0 & 14 & 19 \\
\hline Arterial dilatation & 0 & 25 & 25 & 12 & 17 & 17 & 43 & 83 & 43 & 46 \\
\hline Increased light reflex & $\mathbf{0}$ & 25 & 12 & 12 & 12 & 50 & 57 & 100 & 57 & 65 \\
\hline Granular nerve fibre layer & 0 & 0 & 0 & 0 & 0 & 83 & 100 & 100 & 86 & 92 \\
\hline Peripapillary halo & 0 & 0 & 0 & 0 & 0 & 83 & 100 & 100 & 86 & 92 \\
\hline
\end{tabular}

*The actual number of eyes included in analysis (per subgroup) may differ-see text.

† Two eyes (from the same animal) showed sludging of blood in narrowed vessels-see text. 


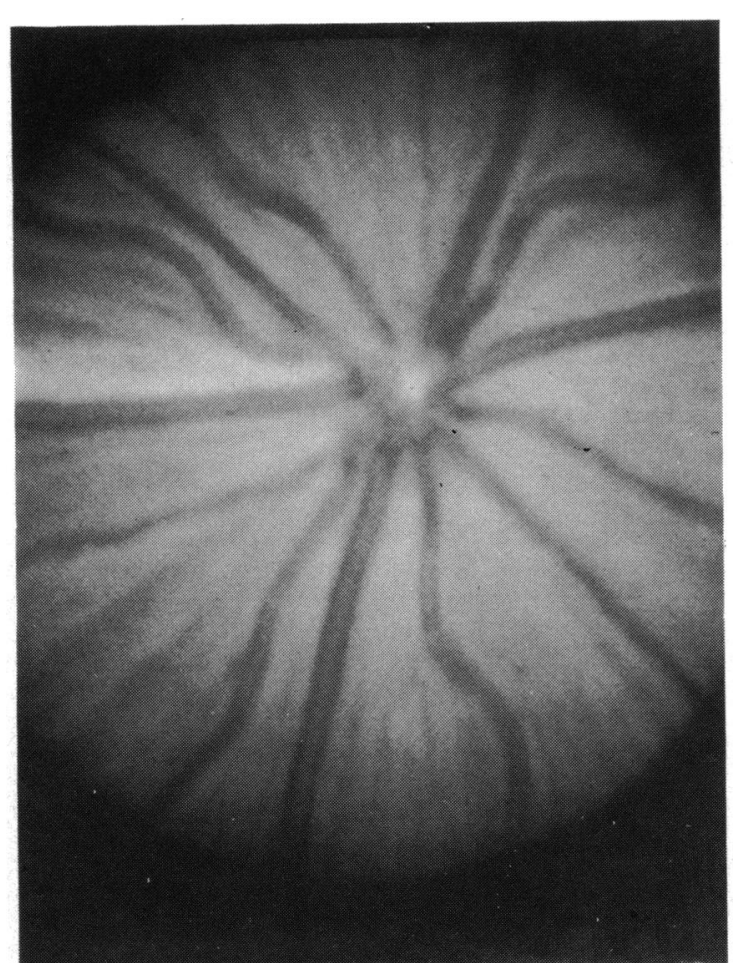

Fig. 1a

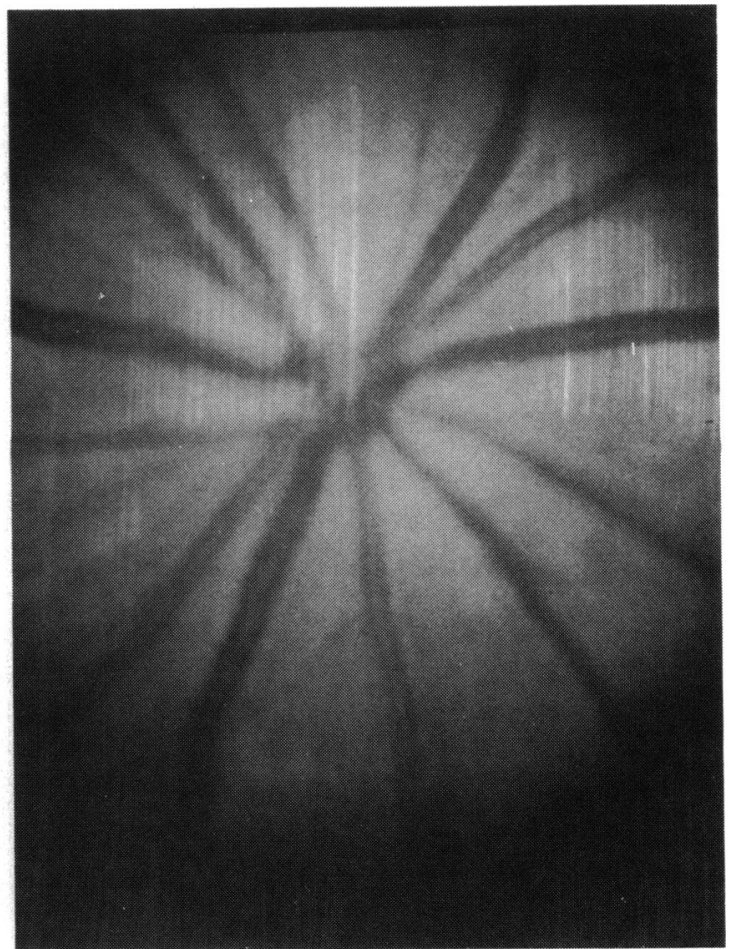

Fig. 1b

Fig. 1 Serial fundus photographs of a rat. (a) Before bilateral ligation (baseline). (b) Two days following bilateral ligation showing dilated veins, straightened arteries, retinal whitening along the venous distribution, and blurring of the optic disc. (c) Prior to ligation removal (12 weeks) showing an increased arterial light reflex, peripapillary halo, and granular-appearing nerve fibre layer. (d) Prior to sacrifice (16 weeks) showing changes as in Fig. 1c.

anaesthetised with pentobarbitone sodium (Nembutal) ( $40 \mathrm{mg} / \mathrm{kg})$ and ketamine $(18 \mathrm{mg} / \mathrm{kg})$ delivered intraperitoneally. The pupils were dilated with a topical $2 \%$ phenylephrine $/ 0 \cdot 2 \%$ atropine solution.

For fluorescein angiography an incision was made in the ventral flank of the animal and the deep femoral vein isolated and cleaned. Two 4-0 silk sutures were placed around the vein, with the more distal one securely tied. A small incision was made in the vein between the 2 sutures and a heparinised 22-gauge catheter threaded through the opening and advanced approximately $1 \mathrm{~cm}$. The more proximal suture was tied around both vein and catheter. The animal was then placed on a special mounting platform.

Fundus photography was performed with a Zeiss Fundus Flash-1 camera and Ektachrome 64 film. Three photographs of each eye were taken. Fluorescein angiography was then performed with $0.5 \%$ $\mathrm{NaFl}$, standard fluorescein filters and Tri-X film. The fluorescein dye was injected within 1 second and manually operated photography begun, with photos taken at approximately 1 -second intervals for the first
15 seconds. A photograph was next taken at 60 seconds and then at 1-minute intervals until 6 minutes had elapsed. The animal was turned and photographs taken of the other eye from 8-20 minutes at 1-minute intervals. Photography of the eye on the side of the ligation or sham procedure (groups $A$ and $C_{1}$ ) or on the side of the planned ligation removal or sham removal procedure (groups B and $\mathrm{C}_{2}$ ) was performed during the first 6 minutes of angiography throughout the experiment. On completion of fluorescein angiography the catheter was removed and the proximal suture secured to prevent bleeding. The skin was closed with 4-0 silk sutures.

For carotid artery ligation an incision was made in the midline of the neck and the right and/or left common carotid artery isolated and cleaned. A single 4-0 silk suture was placed around the carotid and securely tied $1 \mathrm{~cm}$ proximal to the point of bifurcation of the artery. The neck was sutured closed.

Immediately prior to sacrifice the eyes were enucleated and preserved in 5\% formalin for later study. 


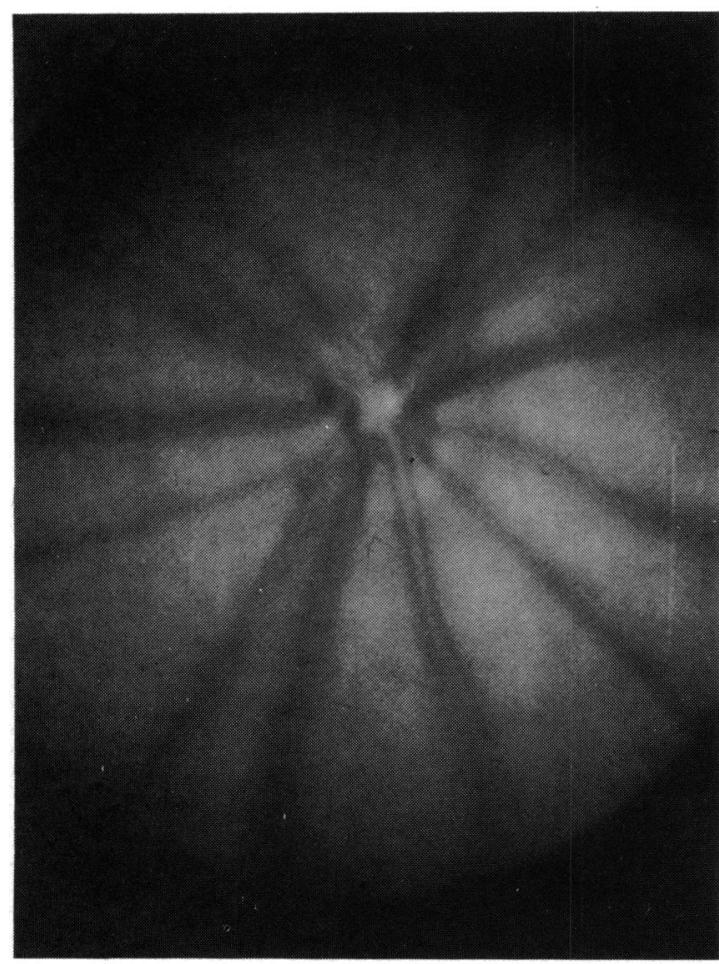

Fig. 1c

\section{Results}

\section{FUNDUS PHOTOGRAPHY}

The fundus photographs of each eye were sequentially arranged, coded by one investigator, and evaluated by another. Abnormalities were determined by comparison of baseline to subsequent photographs. A number of animals developed significant degrees of corneal clouding and cataract formation during the experiment which prevented clear fundus photos from being taken. Only those photographs which were detailed enough to interpret were included in our analysis.

\section{Bilateral ligation (group B)}

Figs. 1-4 demonstrate the marked retinal changes which occur after bilateral common carotid artery ligation. Two days following this operation all eyes from group B animals revealed at least three 'early' fundus abnormalities including dilatation and tortuosity of retinal veins $(92 \%)$; straightening of retinal arteries (92\%); blurring and swelling of the optic disc (disc oedema) (85\%); and varying degrees of retinal whitening $(85 \%)$, primarily along the venous distribution (Figs. 1b, 2). Table 2 summarises the specific fundus changes by subgroup. One animal

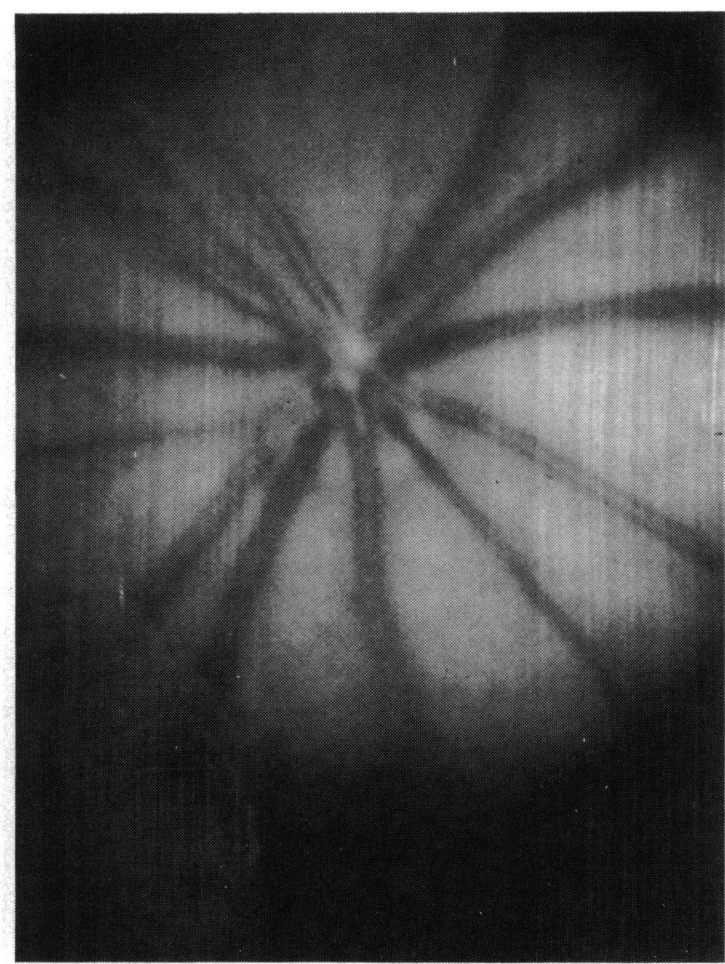

Fig. 1d

( 2 eyes) exhibited a unique fundus pattern in which there was apparent sludging of blood in narrowed retinal vessels, accompanied by severe disc oedema and retinal whitening (Fig. 3).

At the time of ligation removal $(1,4$, or 12 weeks after the ligation procedure, depending on the subgroup-Table 1), all eyes showed resolution of disc oedema and retinal whitening. Furthermore $95 \%$ of these eyes had at least one of the following 'late' fundus abnormalities: narrowed retinal veins; irregular calibre of retinal arteries with an increased light reflex over the vessels; a granular nerve fibre layer (normally striated in appearance); and a peripapillary 'halo' (Fig. 1c). The latter 3 fundus changes were observed more frequently in the eyes of those animals subjected to a longer period of bilateral ligation (Table 2). Subgroup B-IV animals, which did not have their ligations removed, were not studied at this point.

Immediately prior to sacrifice $96 \%$ of all eyes showed at least one of the 'late' fundus abnormalities described above (Figs. 1d, 4). In all subgroups the increased light reflex, granular nerve fibre layer, and peripapillary halo, when present at the time of ligation removal, became more pronounced. Further- 


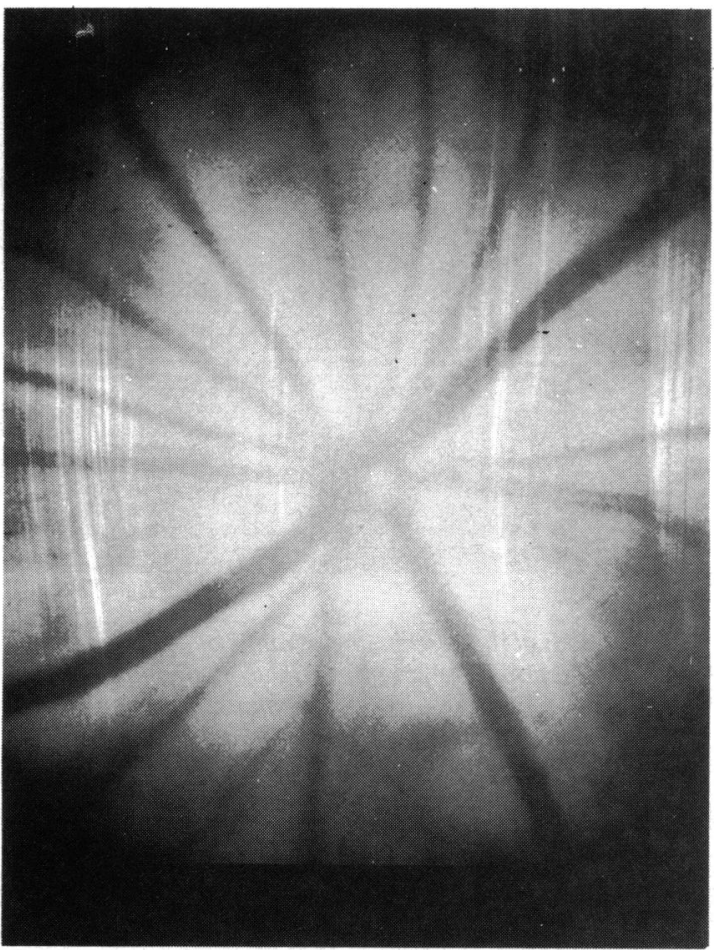

Fig. 2 Another example of marked venous dilation, retinal whitening, and disc oedema, 2 days following bilateral ligation.

more, the latter 2 findings were observed in $92 \%$ of the eyes studied (Table 2 ).

\section{Unilateral ligation (group A)}

Two days following this procedure only 2 eyes $(8 \%)$ from group A animals showed any 'early' fundus abnormalities (Table 2). Retinal venous dilatation and arterial straightening, when they occurred, were less severe than in group B eyes. Disc oedema and retinal whitening were not observed in any eyes.

Immediately prior to ligation removal (subgroup A-IV excluded), only $19 \%$ of eyes demonstrated 'late' fundus changes, including venous narrowing, arterial dilatation, and an increased light reflex over the retinal arteries. Alterations in the appearance of the striated nerve fibre layer and development of a peripapillary halo were not observed (Table 2).

At the time of sacrifice only dilatation of retinal arteries in 4 eyes (17\%) and an increased light reflex in 3 eyes (12\%) were found. Again no halo or nerve fibre layer granularity was noted.

There was no correlation between the fundus abnormalities in the eyes of unilaterally ligated animals and the side on which the ligation was performed.

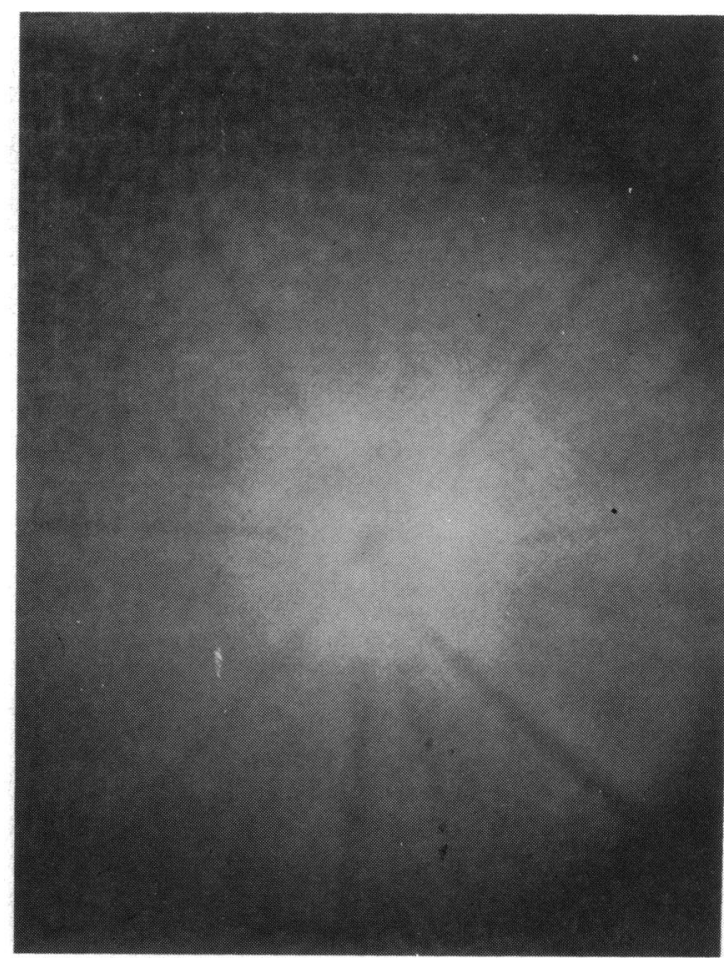

Fig. 3 Sludging of blood in narrowed vessels, disc oedema, and retinal whitening, 2 days after bilateral ligation.

\section{Controls (group C)}

Narrowing of the retinal arteries was found in 2 eyes (8\%) of the control animals 2 days following the initial sham operation, and this constituted the only change seen. A similar change was occasionally noted in the eyes of other animals (all groups) while focusing the camera (before photographs were taken) if the eyeball had been accidentally 'proptosed' when the eyelids were spread in an attempt to obtain a clearer view of the fundus.

Late fundus abnormalities were not observed in the eyes of control animals.

\section{FLUORESCEIN ANGIOGRAPHY}

The fluorescein angiograms of each animal were sequentially arranged, coded by one investigator, and interpreted by another. Again, abnormalities were determined by comparison of baseline to subsequent angiograms. We defined 'delay' as a greater than 2 -second increase in the time between the initial appearance of fluorescein in the retinal arteries and complete filling of the retinal veins, when compared with the individual animal's baseline angiogram. During angiography the camera would occasionally malfunction, leaving gaps in some angiograms. This precluded full evaluation in some animals. 


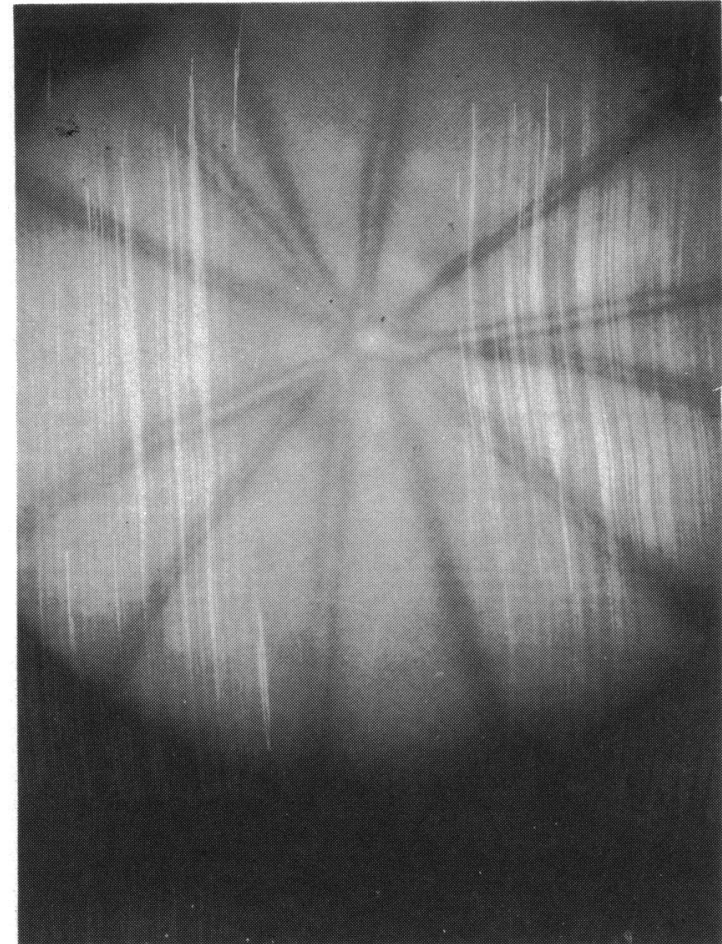

Fig. 4 Increased arterial light reflex, granular nerve fibre layer, and a prominent peripapillary halo, 16 weeks after bilateral ligation.

\section{Bilateral ligation}

All animals showed abnormalities by fluorescein angiography 2 days after the initial operation, including at least one of the following: hyperfluorescence of the disc preceding retinal arterial filling (93\%); venous dilatation (87\%); disc oedema $(80 \%)$; disruption of the retinal capillary bed pattern (73\%); and peripapillary staining/leakage occurring after 60 seconds $(100 \%)$ (Table 3 ). In addition there was a marked delay in the rate of retinal arterial and venous filling in $100 \%$ of the animals. Figs. 6a-f depict the above changes from baseline (Figs. 5a-d) which occurred 2 days following bilateral ligation.

The early hyperfluorescence of the disc, venous dilatation, disc oedema, and late peripapillary staining/leakage were absent from all angiograms at the time of ligation removal. The retinal capillary pattern, however, remained coarse, and the rate of arterial and venous filling continued to be delayed in most animals, although some improvement was noted. In about half the animals an irregular vessel calibre, patchy choroidal pattern, and a peripapillary 'halo' were observed. The angiograms performed immediately prior to enucleation and sacrifice revealed similar findings, although vessel calibre

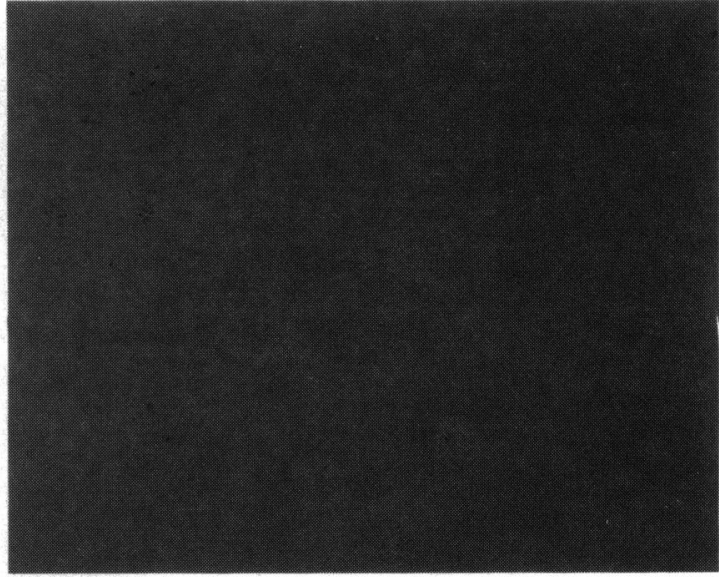

Fig. 5a

Fig. 5 Retinal fluorescein angiographs of a rat prior to bilateral ligation (baseline). (a) At the onset of the arterial phase. (b) During the early venous phase, showing laminar flow in the retinal veins and a sharp capillary bed pattern. (c) At the time of complete venous filling (late venous phase). (d) At approximately 2 minutes after fluorescein injection.

became normal. Figs. 7a-f show these late fluorescein angiographic changes.

\section{Unilateral ligation}

Venous dilatation and a mild delay in the rate of retinal arterial and venous filling were noted in $38 \%$ of the animals 2 days following unilateral ligation. These were the only changes noted in the angiograms at that time. Immediately prior to both ligation

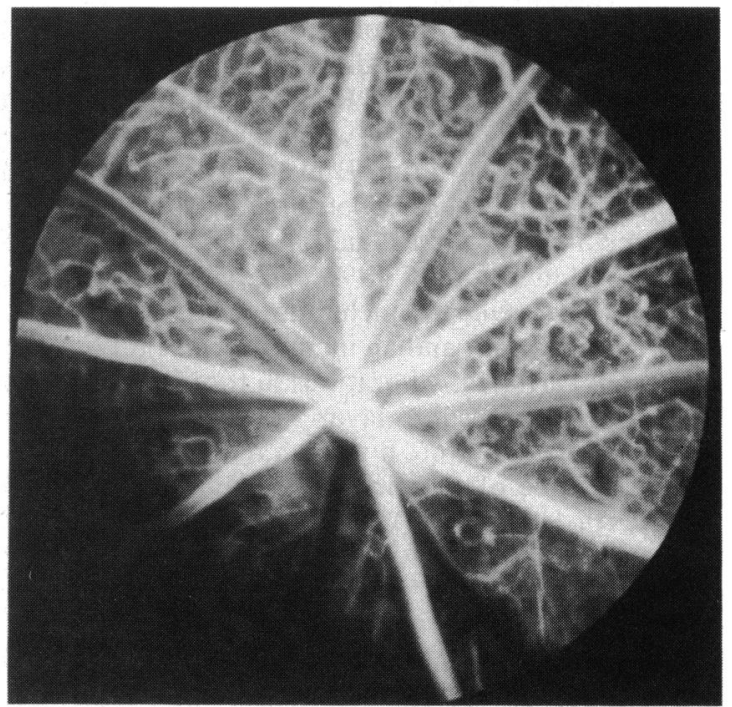

Fig. $5 b$ 


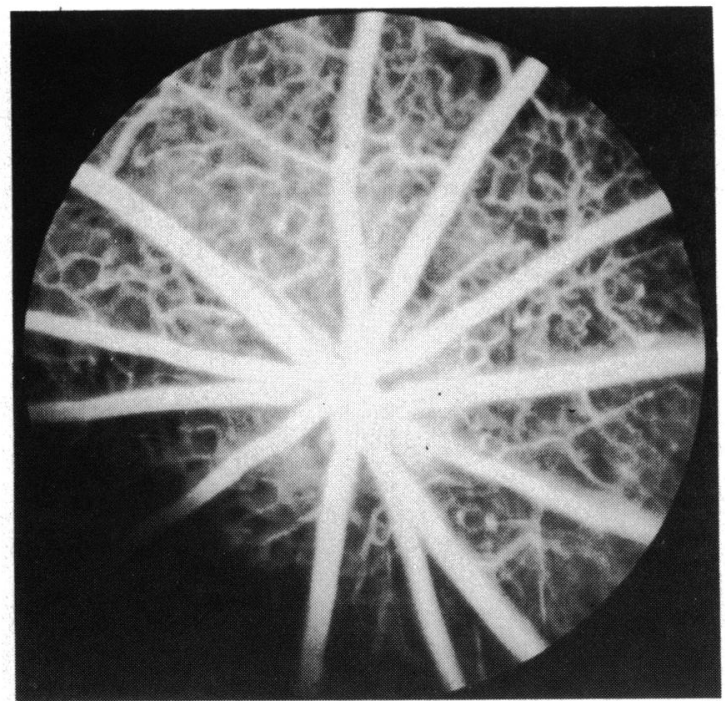

Fig. 5c

removal and sacrifice irregularities in vessel calibre were noted in one animal. In most cases the delays in the rate of filling persisted throughout the study. Only one animal $(9 \%)$ showed capillary disruption at the time of ligation removal, while 2 animals (13\%) showed this abnormality just prior to sacrifice. Table 3 summarises these findings.

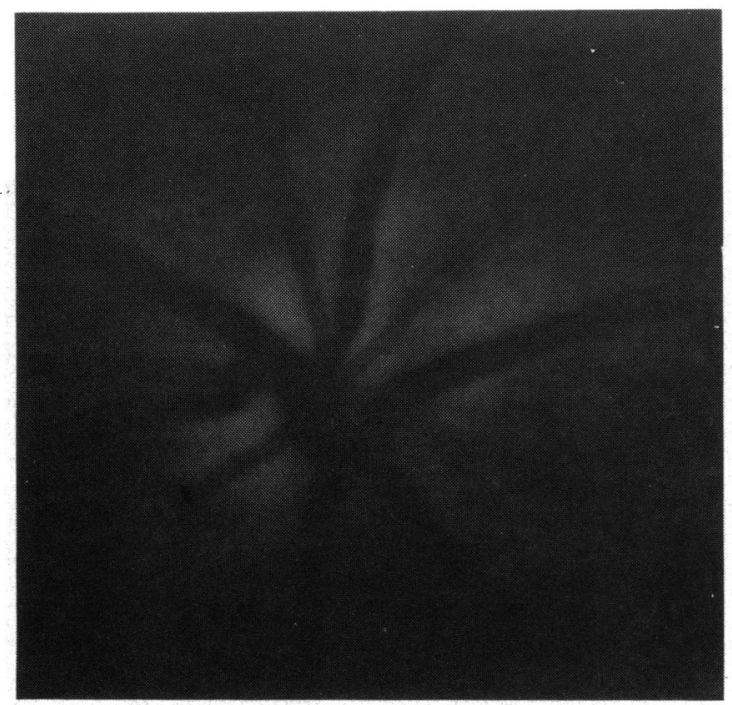

Fig. 6a

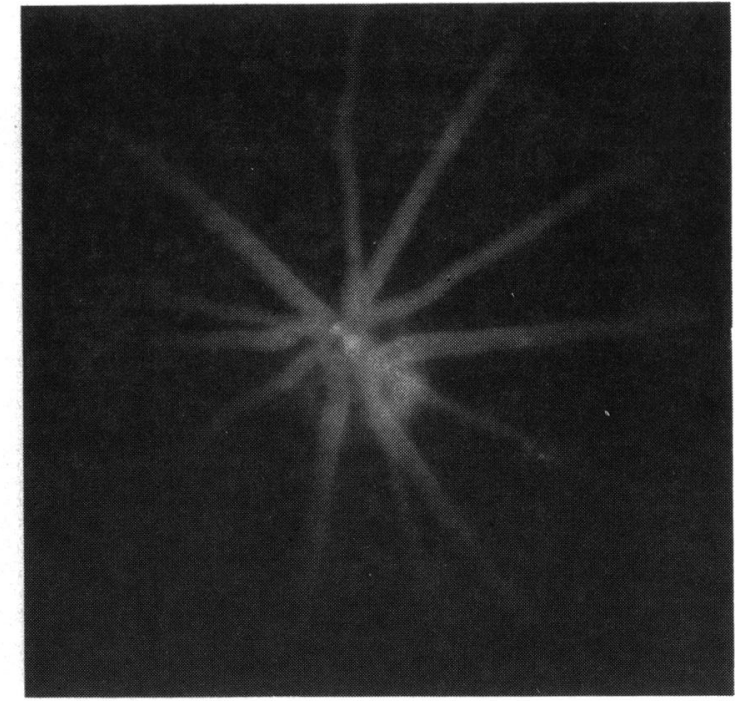

Fig. 5d

\section{Controls}

Control animals showed no abnormalities by angiography.

The averaged time intervals between the phases of fluorescein passage through the retinal circulation are depicted for all 3 experimental groups (Table 4). Since both initiation of photography and injection of

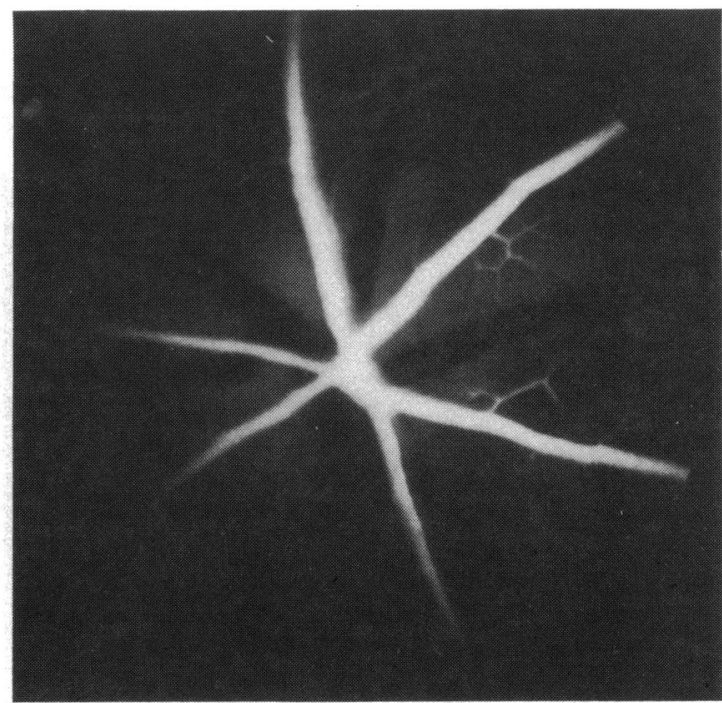

Fig. 6b

Fig. 6 Fluorescein angiographs of the same rat as in Fig. 5, 2 days following bilateral carotid artery ligation. (a) Prior to the onset of the arterial phase showing hyperfluorescence of the disc. (b) After the onset of the arterial phase. (c) At the onset of the early venous phase, showing disc oedema. (d) Later, during venous filling, revealing a coarse capillary pattern. (e) At the completion of the late venous phase. (f) Approximately 2 minutes after fluorescein injection showing disc leakage. Note the delay in all phases of retinal arterial and venous filling when compared with baseline (Figs. 5a-d). 


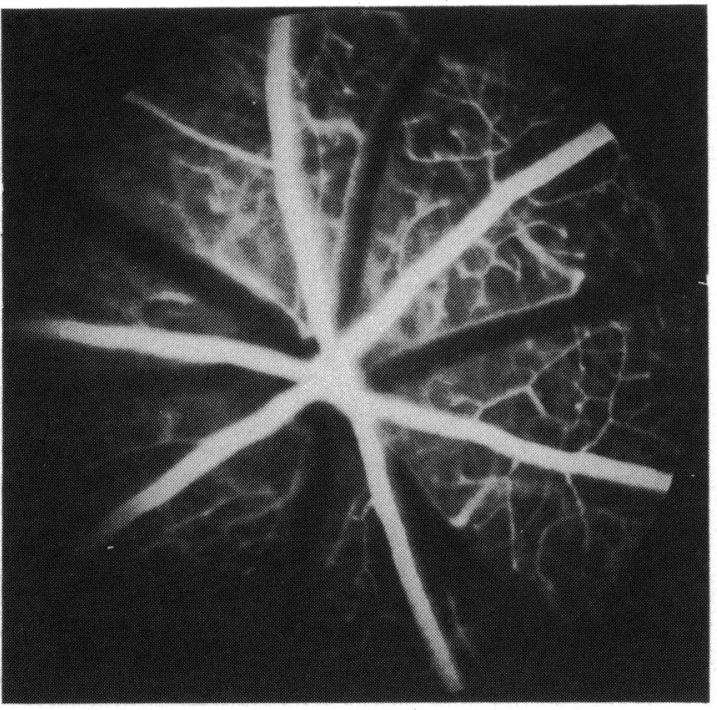

Fig. 6c

fluorescein were manually performed, the exact moment of injection and onset of photography could not be precisely synchronised. For this reason the elapsed times between injection of fluorescein and the first appearance of dye in the arteries were not used for comparison study. The exact moments at which fluorescein dye first appeared in the retinal arteries (arterial phase), laminar flow of dye towards the disc developed in the retinal veins (early venous phase), and complete filling of the veins (late venous phase) occurred were often not seen on the angiograms when taking photographs at the rate of one per

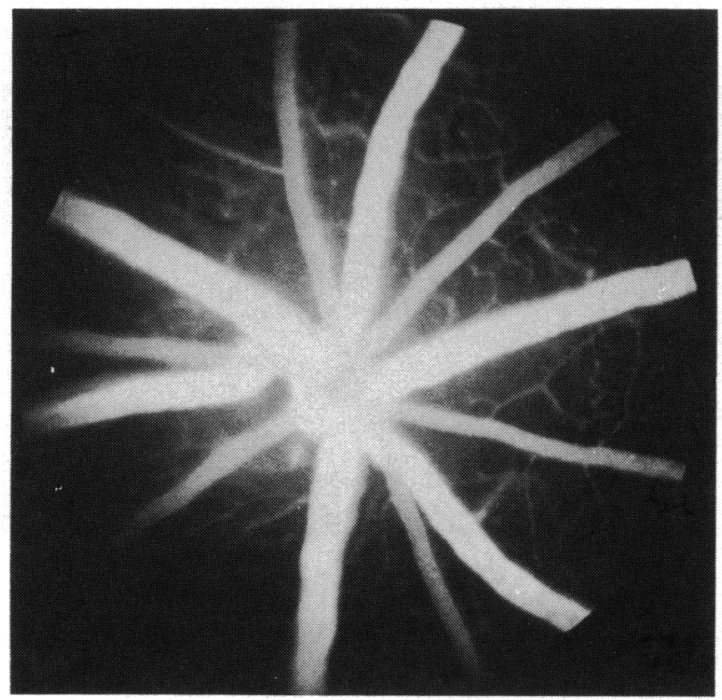

Fig. 6e

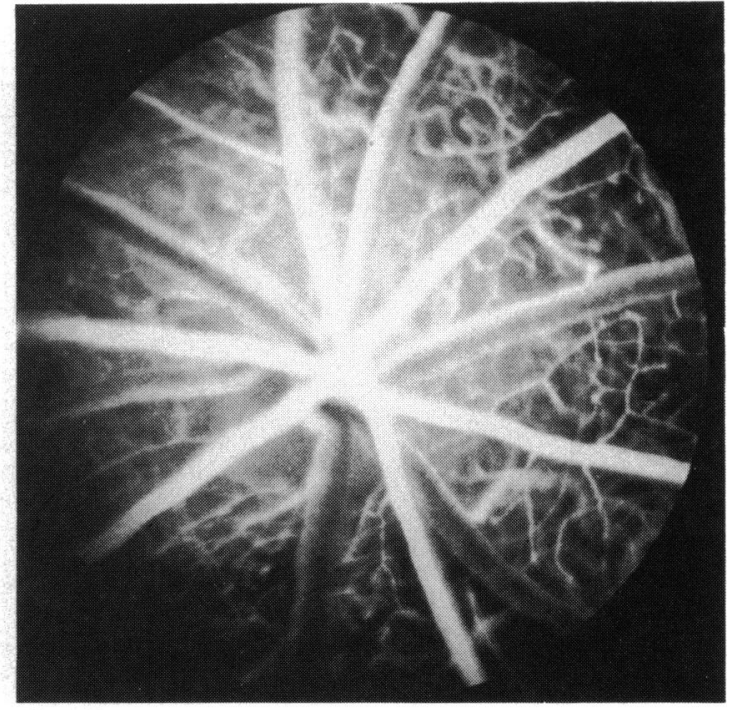

Fig. 6d

second. Therefore the time recorded on the photograph immediately following the event was used in the determination of the intervals between phases. The timing between photographs was not automatic and varied from 0.7 to 1.1 seconds. Thus, only averaged time intervals for each group are displayed.

Analysis of the group B angiograms revealed a marked delay between all phases of fluorescein passage through the retinal circulation when compared with their own baselines as well as with the controls at all stages in the experiment (Table 4). This delay lessened as the experiment progressed, with the

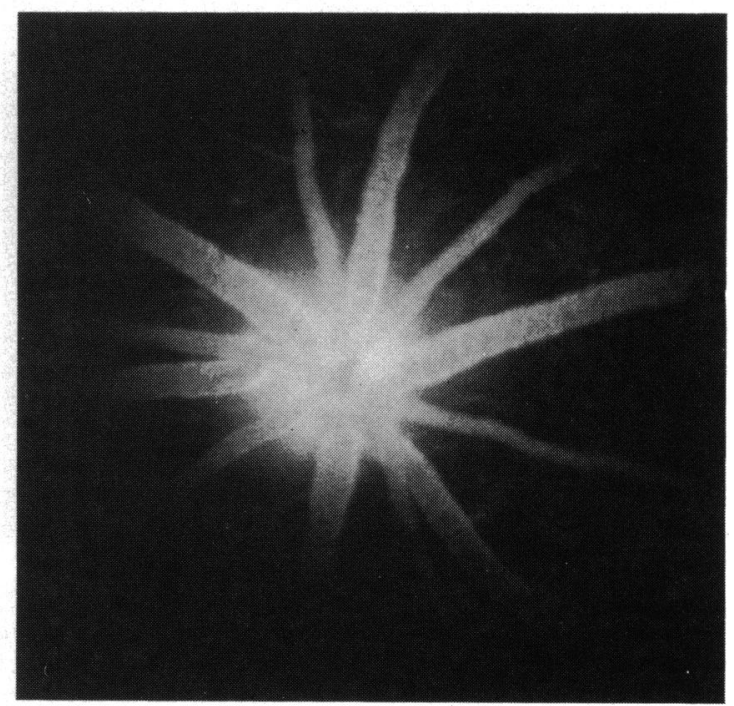

Fig. $6 f$ 


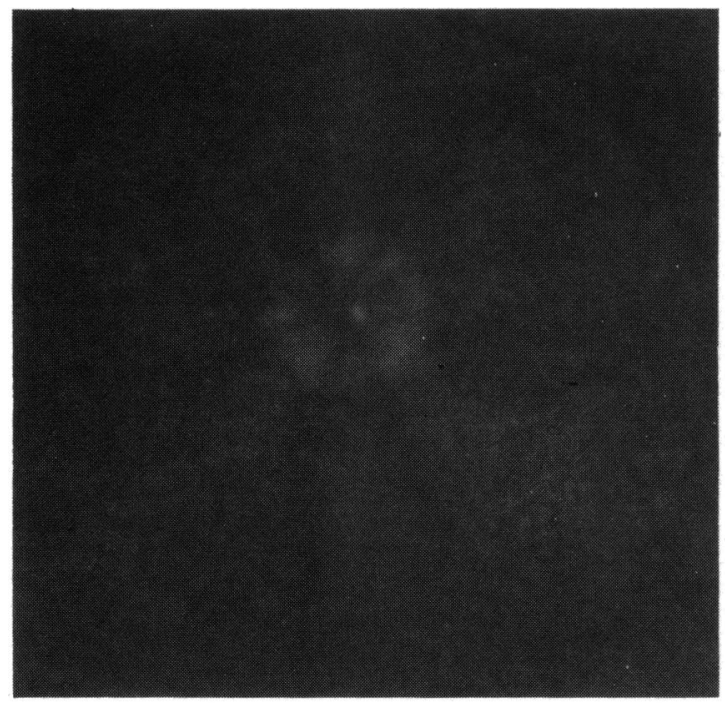

Fig. $7 \mathrm{a}$

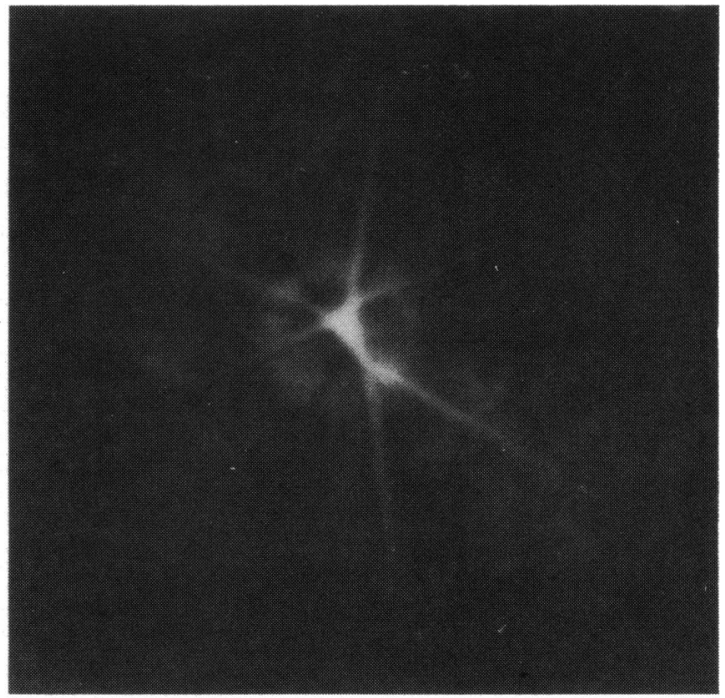

Fig. $7 b$

Fig. 7 Fluorescein angiographs of the same rat as in Figs. 5 and 6, 16 weeks after bilateral ligation. (a) Prior to the onset of the arterial phase showing a peripapillary halo. (b) At the onset of the arterial phase. (c) Later in the arterial phase, revealing a patchy choroidal pattern. (d) At the onset of the early venous phase, showing a disrupted capillary bed pattern. (e) During the late venous phase. (f) At approximately 2 minutes after fluorescein injection.

decrease in the time interval between the arterial and early venous phases appearing to be greater than that for the interval between the early and late venous phases. Examination of group A angiograms revealed a much milder delay in all phases when compared in the same manner.

\section{Discussion}

We have produced a characteristic fundus appearance following acute bilateral common carotid artery ligation in mature rats which resembles the human picture of carotid occlusive disease. This retinopathy,

Table 3 Percentage of animals showing specific abnormalities by fluorescein angiography

\begin{tabular}{|c|c|c|c|c|c|c|c|c|c|c|}
\hline & \multicolumn{5}{|c|}{ Unilateral ligation } & \multicolumn{4}{|c|}{ Bilateral ligation } & \multirow{2}{*}{ Total } \\
\hline & $I$ & II & III & $I V$ & Total & $I$ & $I I$ & III & $I V$ & \\
\hline \multicolumn{11}{|l|}{ Two days following ligation: } \\
\hline Early disc hyperfluorescence & 0 & $\mathbf{0}$ & 0 & 0 & 0 & 100 & 100 & 100 & 75 & 93 \\
\hline Filling delay* & 25 & 50 & $33+$ & 33 & 36 & 100 & 100 & 100 & 100 & 100 \\
\hline Venous dilatation & 0 & 25 & 25 & 0 & 13 & 67 & 100 & 100 & 75 & 87 \\
\hline Capillary pattern disruption & 0 & 0 & $\mathbf{0}$ & 0 & 0 & 33 & 100 & 75 & 75 & 73 \\
\hline Disc oedema & 0 & 0 & $\mathbf{0}$ & 0 & 0 & 67 & 100 & 100 & 50 & 80 \\
\hline Late peripapillary leakage & 0 & 0 & 0 & 0 & 0 & 100 & 100 & 100 & 100 & 100 \\
\hline \multicolumn{11}{|l|}{ Prior to ligation removal: } \\
\hline Filling delay* & 0 & 67 & 50 & - & 36 & 100 & 75 & 75 & - & 83 \\
\hline Irregular vessel calibre & $\mathbf{0}$ & 0 & 25 & - & 9 & 50 & 50 & 50 & - & 50 \\
\hline Capillary pattern disruption & 0 & 0 & 25 & - & 9 & 50 & 100 & 100 & - & 83 \\
\hline Patchy choroidal pattern & 0 & 0 & 0 & - & 0 & 75 & 25 & 75 & - & 58 \\
\hline Peripapillary halo & 0 & 0 & 0 & - & 0 & 75 & 50 & 50 & - & 58 \\
\hline \multicolumn{11}{|l|}{ Prior to sacrifice: } \\
\hline Filling delay* & 0 & $67 \dagger$ & 50 & 33 & 36 & 33 & 100 & 100 & 100 & 86 \\
\hline Irregular vessel calibre & 0 & 0 & 0 & 33 & 7 & $\mathbf{0}$ & 0 & 0 & 0 & 0 \\
\hline Capillary pattern disruption & 0 & 0 & 50 & 0 & 13 & 67 & 100 & 100 & 75 & 86 \\
\hline Patchy choroidal pattern & $\mathbf{0}$ & 0 & 0 & 0 & 0 & 33 & 50 & 67 & 75 & 57 \\
\hline Peripapillary halo & 0 & 0 & 0 & $\mathbf{0}$ & 0 & 67 & 100 & 67 & 50 & 71 \\
\hline
\end{tabular}

* See text.

†Only 3 of 4 angiograms evaluated (camera malfunction). 


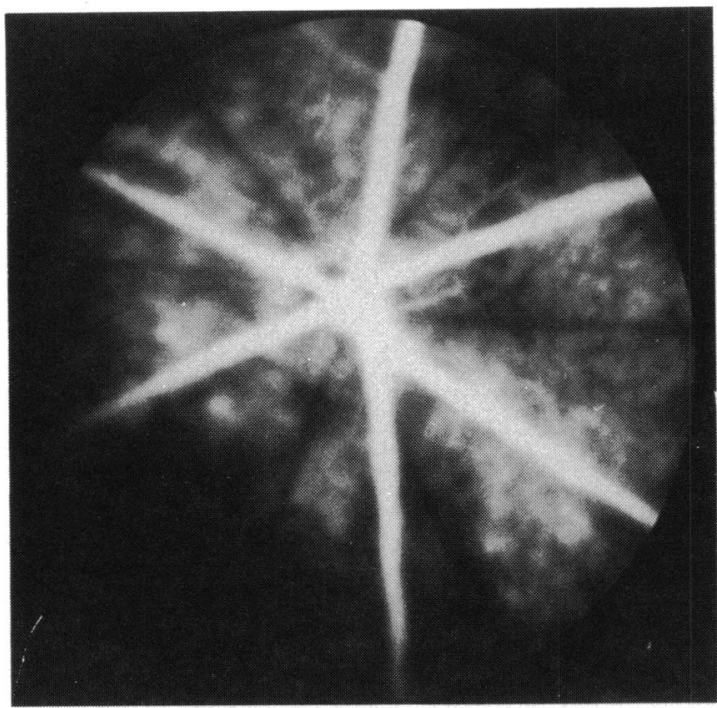

Fig. 7c

which was observed 2 days following bilateral ligation, consisted of dilatation and tortuosity of retinal veins, blurring and swelling of the optic disc, retinal whitening (possible cotton-wool spots), and straightening of retinal arteries. Retinal haemorrhages, microaneurysms, and neovascularisation were not found. This retinopathy was not observed in the eyes of unilaterally ligated animals.

Fluorescein angiograms of bilaterally ligated animals also showed a consistent pattern when angiography was performed 2 days following ligation. Hyperfluorescence of the disc (preceding retinal arterial filling), delay in the rate of retinal arterial and venous filling, venous dilatation, disc oedema, disruption of the retinal capillary bed pattern, and late peripapillary staining/leakage were observed. Angiography of unilaterally ligated animals showed only venous dilation and an occasional delay in retinal arterial and venous filling.

The characteristic retinopathy, as demonstrated by fundus photography and fluorescein angiography 2

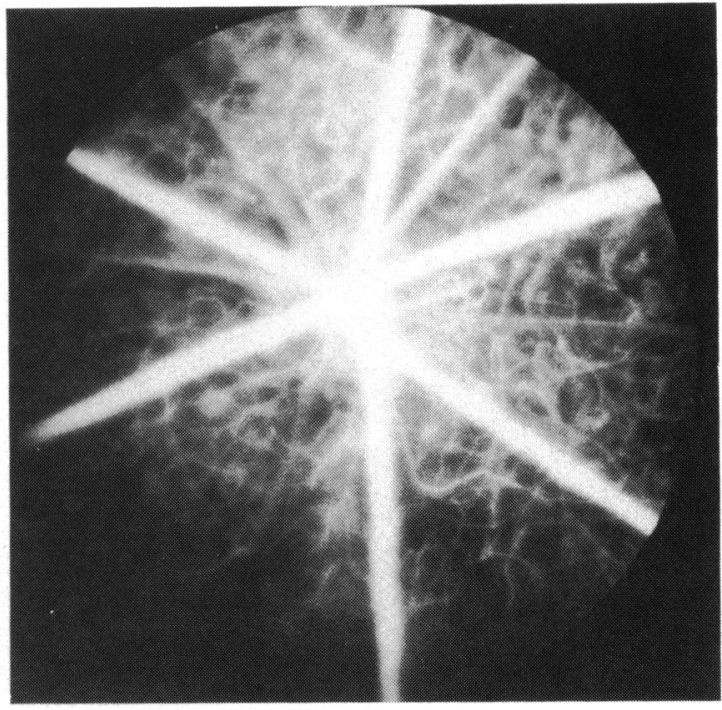

Fig. 7d

days following acute bilateral ligation, was seen to resolve spontaneously by 1 week. These initial abnormalities were replaced by fundus changes of a more 'structural' nature. The granular-appearing nerve fibre layer and peripapillary 'halo' probably represent destruction of the optic nerve fibres and focal dropout of the pigment epithelium respectively. Fluorescein angiographic demonstration of late choroidal and capillary damage and a marked peripapillary 'halo' further support this hypothesis. Late sequelae of unilateral ligation were essentially restricted to changes in the retinal vessels.

Fundus photography and fluorescein angiography were both effective in demonstrating the severe retinal changes following bilateral ligation. However, fluorescein angiography proved to be a more sensitive method for detecting subtle changes in the retinal microvasculature following unilateral ligation.

Our goal was to develop an animal model to study the ocular effects of acute carotid occlusion. The pigmented rat was chosen with an understanding of

Table 4 Averaged time intervals (in seconds) between phases* of fluorescein passage through the retinal circulation

\begin{tabular}{|c|c|c|c|c|c|c|c|c|c|}
\hline & \multicolumn{3}{|c|}{ Arterial-early venous } & \multicolumn{3}{|c|}{ Early venous-late venous } & \multicolumn{3}{|c|}{ Arterial-late venous } \\
\hline & Group C & Group $A$ & Group B & Group C & Group $A$ & Group B & Group C & Group A & Group $B$ \\
\hline Baseline & 0.9 & 0.7 & $0 \cdot 6$ & $1 \cdot 2$ & $1 \cdot 1$ & $1 \cdot 3$ & $2 \cdot 1$ & $1 \cdot 8$ & $1 \cdot 9$ \\
\hline Two days following ligation & 0.9 & $1 \cdot 1$ & $2 \cdot 9+$ & $1 \cdot \overline{3}$ & $2 \cdot 1$ & $4 \cdot 9 \ddagger$ & $2 \cdot 1$ & $3 \cdot 2$ & $7 \cdot 3 \ddagger$ \\
\hline Prior to ligation removal & $0 \cdot 8$ & $1 \cdot 0$ & $1 \cdot 8$ & $1 \cdot 2$ & $2 \cdot 2$ & $3 \cdot 8$ & $2 \cdot 0$ & $3 \cdot 2$ & $5 \cdot 6$ \\
\hline Prior to sacrifice & $0 \cdot 7$ & $1 \cdot 2$ & $1 \cdot 4$ & $1 \cdot 2$ & $2 \cdot 4$ & $3 \cdot 7$ & 1.9 & 3.6 & $5 \cdot 1$ \\
\hline
\end{tabular}

* See text.

† Excluding one animal where arterial filling began after the initial 15 seconds. No accurate time determination could be made

$\ddagger$ Excluding 4 animals where complete venous filling occurred after the initial 15 seconds. No accurate time determination could be made, although the arterial to late venous phase exceeded 10 seconds. 


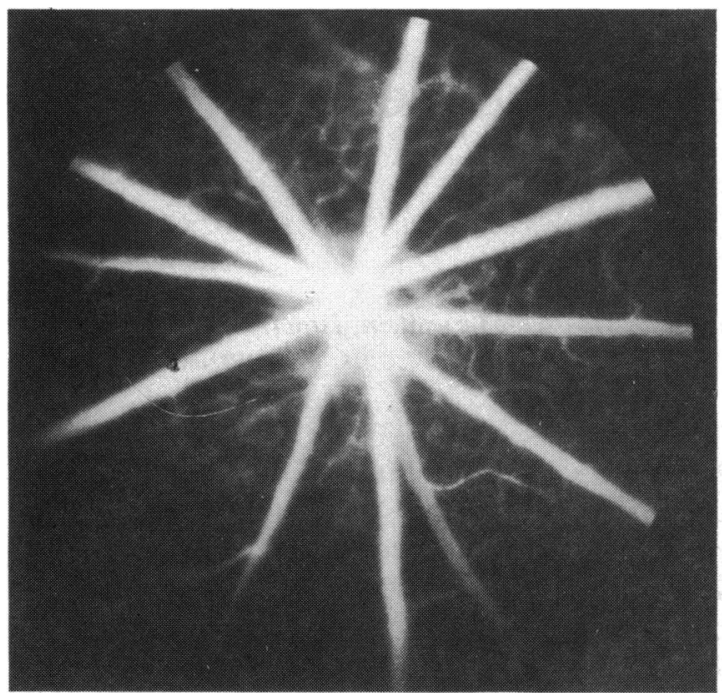

Fig. 7e

its similarities to and differences from human anatomy. While the major vessels in the head and neck of the rat are analogous to those in the human, some differences do exist in the retina of the rat which may preclude the development of retinal haemorrhages, microaneurysms, and neovascularisation seen in some humans with carotid occlusive disease. In addition the venous tortuosity seen in our animals was not of the same severity as that found in the fundi of humans with the disease. This can be explained by the fact that the retinal arteries and veins in the rat, unlike the human, are not bound in a common adventitial sheath. Thus the retinal veins in the rat are free to dilate and lengthen as dictated by haemodynamic requirements. In spite of these differences we have demonstrated that the pigmented rat serves as an inexpensive, reproducible animal model for the study of the retinal sequelae of acute carotid occlusion.

The original protocol for this experiment called for studying the reversibility of the effects resulting from acute carotid occlusion. However, during the ligation removal procedures it was felt that patency of the vessels could not be uniformly re-established due to extensive tissue reaction around the vessel at the site of ligation. Thus, that aspect of the experiment was not considered here. Our coworkers are at present exploring the question of reversibility by a different ligation technique.

We thank Noel Roa, Kenn Kostuk, and Larry Egan for their technical assistance.

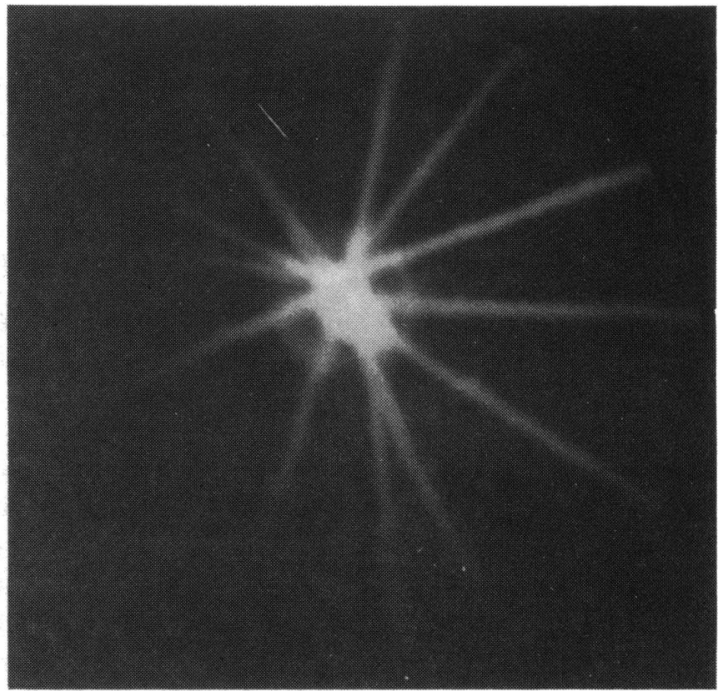

Fig. $7 f$

Supported by an unrestricted research grant from Research to Prevent Blindness.

\section{References}

1 Kearns TP, Hollenhorst RW. Venous-stasis retinopathy of occlusive disease of the carotid artery. Mayo Clin Proc 1963; 38: 304-12.

2 Hollenhorst RW. Ocular manifestations of insufficiency or thrombosis of the internal carotid artery. Trans Am Ophthalmol Soc 1958; 56: 474-506.

3 Swan KC, Raaf J. Changes in the eye and orbit following carotid ligation. Trans Am Ophthalmol Soc 1951; 49: 435-44.

4 Hart CT, Hayworth S. Bilateral common carotid occlusion with hypoxic ocular sequelae. Br J Ophthalmol 1971; 55: 383-8.

5 Kearns TP. Ophthalmology and the carotid artery. Am J Ophthalmol 1979; 88: 714-22.

6 Hedges TR Jr. Ophthalmoscopic findings in internal carotid artery occlusion. Am J Ophthalmol 1963; 55: 1007-12.

7 David NS, Norton EWC, Gass DJ, Sexton R. Fluorescein retinal angiography in carotid occlusion. Arch Neurol 1966; 14: 281-7.

8 Barany E. Influence of local arterial blood pressure on aqueous humor and intraocular pressure. Acta Ophthalmol (Kbh) 1946; 24: $337-65$.

9 Best M, Plechaty G, Harris L, Galin MA. Ophthalmodynamometry and ocular pulse studies in carotid occlusion. Arch Ophthalmol 1971; 85: 334-8.

10 Lavenstein B, Milder B, Winkelman JZ, Zappia RJ, Gay SJ. Retinal and choroidal circulation in rabbits. Arch Ophthalmol 1971; 85: 723-9.

11 Newsom WA, Leverett SD, Kirkland VE. Fluorescein angiography and experimental carotid occludion. Arch Ophthalmol 1968; 80: 377-83.

12 Levine S, Payan H. Effects of ischemia and other procedures on the brain of the gerbil (Meriones unguiculates). Exp Neurol 1966; 16: $255-62$.

13 Slakter JS, Spertus AD, Weissman SS, Henkind P. Retinal vascular changes following selective carotid artery ligation in rats. Invest Ophthalmol Visual Sci 1983; 24 (suppl 1): 290. 